\title{
Labyrinthe
}

$12 \mid 2002$

Numéro 12

\section{Pour le dessin baroque napolitain}

\section{Sophie Harent}

\section{OpenEdition}

Journals

Édition électronique

URL : http://journals.openedition.org/labyrinthe/1359

DOI : $10.4000 /$ labyrinthe.1359

ISSN : 1950-6031

Éditeur

Hermann

Édition imprimée

Date de publication : 30 juin 2002

Pagination : 09-4

Référence électronique

Sophie Harent, "Pour le dessin baroque napolitain », Labyrinthe [En ligne], 12 | 2002, mis en ligne le 15 octobre 2006, consulté le 02 mai 2019. URL : http://journals.openedition.org/labyrinthe/1359 ; DOI : 10.4000/labyrinthe.1359

Ce document a été généré automatiquement le 2 mai 2019.

Propriété intellectuelle 


\title{
Pour le dessin baroque napolitain
}

\author{
Sophie Harent
}

1 « On pourrait avancer l'idée que Naples, dans son esprit même, est trop riche en couleur, trop enchantée par le spectacle de sa propre confusion, pour avoir donné de grands maîtres dans l'art du dessin. À tort ou à raison, dans le passé, les historiens se sont servis d'arguments semblables et leur attitude a causé bien des ravages : le public ignore tout simplement l'existence des dessins napolitains, et les spécialistes ont pour une fois fait cause commune avec leur public. » C'est ainsi que W. Vitzthum définissait en 1963 l'état de mépris et de méconnaissance dans lequel semblait avoir été plongé l'art napolitain. Si depuis une trentaine d'années, les historiens italiens se sont chargés d'apporter un démenti à ces propos pessimistes ${ }^{1}$, en France, Naples et ses artistes constituent toujours un pan oublié des recherches scientifiques menées dans le domaine des arts graphiques. De récentes expositions ont permis de faire le point sur la peinture napolitaine des XVII ${ }^{\mathrm{e}}$ et $\mathrm{XVIII}^{\mathrm{e}}$ siècles, mais, fait significatif, aucune rétrospective n'a été consacrée, en France, au dessin napolitain depuis plus de vingt ans.

2 Ville de contrastes, façonnée par les soubresauts de l'histoire et les caprices de la nature, Naples reste toujours un immense et fascinant terrain de recherche, encore peu ou mal exploré hors des frontières italiennes. Beaucoup de dispersions, peu de certitudes, une indifférence marquée pour l'art napolitain, vulgaire, "sec », "sans force et sans génie ${ }^{2}$ " ont longtemps condamné la capitale méridionale à l'indifférence et au mépris. Négligé au profit d'autres écoles jugées plus nobles et plus fécondes, le foyer napolitain ne semblait pas avoir connu, à partir du XVII ${ }^{\mathrm{e}}$ siècle, plus de deux ou trois artistes éminents. Encore en allait-il plus radicalement des années 1650-1750, où la décadence annoncée des Bourbons trouvait sa plus belle expression dans l'art fade des suiveurs de Francesco Solimena.

3 C'est ainsi que Luca Giordano et Francesco Solimena semblaient résumer à eux seuls l'art napolitain de l'âge «baroque ». Si d'aventure apparaissaient les noms de P. de Matteis, G. del Po, F. de Mura ou C. Giaquinto, ce n'était encore que par opposition ou comparaison dépréciative avec leurs maîtres, tandis que les Vite de'pittori, scultori ed architetti napoletani, rédigées par Bernardo De Dominici dans la première moitié du XVIII ${ }^{\mathrm{e}}$ siècle, source privilégiée sur ces artistes, restaient confinées à un anonymat presque absolu. 
Les précurseurs

4 Roberto Longhi ${ }^{3}$ et Louis Dimier ont été les premiers à s'intéresser de nouveau à l'art napolitain des XVII ${ }^{\mathrm{e}}$ et XVIII ${ }^{\mathrm{e}}$ siècles, après le long silence auquel la critique académique du $\mathrm{XIX}^{\mathrm{e}}$ siècle, méprisant le naturalisme caravagesque et les différentes expressions de la culture « baroque », avait conduit les historiens de l'Italie méridionale.

5 En France, L. Dimier donnait enfin de l'art napolitain, dans deux articles de la revue les Arts publiés en 1908 et 1909, une perception plus nuancée, en réhabilitant successivement L. Giordano, S. Rosa, A. Vaccaro ou M. Stanzione, puis F. Solimena, N. M. Rossi, S. Conca ou bien encore G. del Po. À sa suite, Louis Hautecœur exprimait dès 9, dans la Gazette des beaux-arts, son intérêt pour "Les arts à Naples au XVIII ${ }^{\mathrm{e}}$ siècle ». Le goût de la variété, du pittoresque et de l'exagération, était selon lui inconciliable avec la redécouverte de l'antique qui absorbait alors l'Europe et expliquait la condamnation sans appel dont avait été victime l'école napolitaine.

6 Appelant de ses vœux une nécessaire réhabilitation, Hautecoeur vit ses efforts récompensés dès les années 1930 en Italie par plusieurs expositions, qui demeuraient cependant sans équivalent français. Si la peinture napolitaine retrouvait une place auprès des autres écoles italiennes, l'art graphique demeurait ignoré. Les années soixante allaient sonner le glas de l'oubli et le renouveau de la critique française, tandis que se multipliaient les recherches italiennes sur les archives municipales napolitaines.

7 Faisant suite à L'Art italien $d u X^{X}$ III $^{e}$ siècle, une rétrospective organisée en 1960-1961 au Petit-Palais par Giuseppe Fiocco et plusieurs illustres spécialistes (R. Causa, C. Gnudi, C. Brandi ou V. Moschini... $)^{4}$, W. Vitzthum commençait à entreprendre une enquête fondamentale au cœur des cabinets de dessins du monde entier. Des études jusque-là inconnues étaient pour la première fois exhumées des portefeuilles du Louvre et de quelques musées de province, notamment d'Orléans, où plusieurs annotations portées sur les montages témoignent aujourd'hui encore du passage de l'historien allemand.

Une importante exposition ${ }^{5}$ pouvait alors prendre place à Paris en 1967, et plaider enfin pour la connaissance de ce patrimoine graphique quasiment inédit en France. Pour la première fois, une rétrospective dévoilait quelques exemples du dessin napolitain $\mathrm{du} \mathrm{XVI}^{\mathrm{e}}$ $\mathrm{au} \mathrm{XVIII}^{\mathrm{e}}$ siècle présents dans les collections du musée du Louvre. Un premier regroupement stylistique et un bilan sur les collectionneurs français étaient ainsi proposés.

9 L'embellie fut pourtant de courte durée, et tandis que la peinture napolitaine continuait à bénéficier de découvertes importantes et d'expositions, le dessin napolitain retournait à sa léthargie. En dépit de deux rétrospectives, en 1983, la première au palais de Tokyo, la seconde à l'École nationale supérieure des beaux-arts (consacrée aux dessins des collections publiques napolitaines), qui ont permis de remettre les productions graphiques méridionales sur le devant de la scène critique ${ }^{6}$, le vaste travail de recensement et d'identification entrepris plusieurs dizaines d'années plus tôt par W. Vitzthum (disparu prématurément en 1971) ne trouvait pas de successeur véritable en France.

Les raisons d'une infortune

10 Longtemps jugé sans délicatesse et incapable de s'élever au-dessus de la chronique locale, le dessin napolitain reste aujourd'hui encore oublié et relégué au dernier rang des écoles italiennes. Bien des préjugés continuent à entretenir le mythe d'artistes indifférents aux 
productions graphiques, dont Caravage avait fourni l'exemple archétypique, lui qui, on le sait, tenait le dessin en grand mépris.

11 Si certains ont parfois contribué à amplifier la légende (Paolo de Matteis construisait patiemment son image publique et sa renommée artistique en se débarrassant de ses dessins), la vision négative qui entache le dessin napolitain provient en réalité d'un malentendu fondamental, issu des préjugés développés par le système académique. Deux conceptions distinctes ont en effet été trop souvent confondues : la notion abstraite de dessin, au sens où l'entend Vasari, et le dessin vivant tel que le pratiquent la plupart des artistes napolitains. On ne peut douter en effet que le travail préparatoire ait été une étape essentielle pour ces créateurs. Bien particulièrement précieux pour le peintre, synonyme de sécurité et de gain de temps, il est une source à laquelle l'artiste peut sans cesse puiser le renouvellement de son inspiration. À travers lui s'exprime un monde intérieur riche et complexe, qui nous éclaire à la fois sur sa personnalité, son statut ou ses ambitions. Passage obligé pour l'élaboration des tableaux de chevalet et des grandes compositions peintes, le dessin manifeste également l'étroite communauté d'esprit dans laquelle travaillent ces artistes. Les thèmes abordés (scènes religieuses inspirées par la Contre-Réforme, références littéraires et philosophiques à l'Arcadie de Sannazaro...), les références invoquées (école des Carrache, Raphaël, Lanfranco...), l'homogénéité artistique des techniques employées, traduisent un intérêt commun pour les études graphiques. Mais un intérêt particulier, où le trait à la pierre noire ou à la plume ne peut se détacher d'un goût immodéré pour la couleur.

Or, la critique des productions napolitaines avait reposé en grande partie sur une opposition de principe à des peintres trop peu soucieux de règles et de théorie. Les Français, en effet, opposaient toujours le «feu " (l'imagination créatrice, la capacité de couvrir de grandes surfaces et de les peupler de nombreux personnages, la vigueur du métier pictural), et la « correction » (correction du dessin et, plus largement, fidélité à la nature observée ou filtrée à travers les interprétations de l'Antiquité, de Raphaël et des Carrache). Accusés d'être d'abord des praticiens manquants de noblesse et d'élégance, travaillant avec une facilité et une rapidité suspectes, les peintres napolitains prêtaient particulièrement le flanc à de tels reproches, aggravés par un goût marqué pour les tons sombres (les ombres sont toujours jugées trop noires et méplates) et un manque d'attention au dessin, incompatibles disait-on avec la marque du véritable génie.

Encore soumise par ailleurs à un apprentissage considéré comme archaïque dans l'atelier d'un maître, la formation reçue par les jeunes peintres à Naples parut longtemps fort éloignée des nouvelles expériences académiques françaises, florentines, et surtout romaines. Le voyage à Rome était vu comme une fuite nécessaire pour les artistes les plus prometteurs (P. de Matteis, C. Giaquinto, S. Conca et même l'Abbate Ciccio, F. Solimena), symptôme caractéristique des insuffisances de l'enseignement et par voie de conséquence, de l'art napolitain. Quand l'académie succéda (tardivement, sous le règne de Charles III) à l'atelier, mettant fin à l'ancien système des corporations et consacrant ainsi le triomphe d'une organisation étatisée de l'enseignement des beaux-arts, Naples suscita peu à peu un regain d'intérêt de la part de ses plus farouches détracteurs.

Pourtant la fantaisie de ces artistes insolites, si étrangers au "disegno " vasarien, excellents décorateurs, goûtant aux formes puissantes et aux compositions foisonnantes, ne laissèrent pas les collectionneurs indifférents. Ces amateurs, aristocrates, lettrés, membres de la bourgeoisie, ont été des mécènes particulièrement généreux et exigeants pour les artistes parthénopéens : Carafa, Pietro Ottoboni, A. Harrach, Shaftesbury, Louis 
XIV, le comte d'Estrées ont contribué à asseoir la réputation de Solimena et des peintres de son cercle. Forts d'une renommée internationale qui leur permet d'obtenir des commandes prestigieuses à l'étranger, ces artistes occupent une place éminente dans l'entourage des grands et au sein des cercles intellectuels et artistiques européens.

Dans le concert de ces relations entre "mécènes et peintres", Naples et la France entretiennent des relations complexes, faites d'échecs et d'incompréhension, mais aussi d'admiration et d'émerveillement. Échanges, recherches communes, renforcées par les liens dynastiques entre les cours Bourbons et la multiplication des voyages d'artistes, ne vont cesser de s'accroître. Plusieurs "Français» avaient travaillé dans la cité parthénopéenne depuis le début du XVII ${ }^{\mathrm{e}}$ siècle (S. Vouet, C. Mellin, Didier Barra, François de Nomé). Les jeunes pensionnaires de l'Académie de France à Rome feront du voyage à Naples une étape nécessaire au cours de leur séjour italien. Les copies dessinées de fresques, tableaux et études parthénopéennes, exécutés par F. Boucher, J.-H. Fragonard, J.-R. Ango, et plus tard Doyen ou Pajou, en sont sans doute l'une des plus belles expressions.

Les dessins baroques napolitains des collections publiques françaises

16 Ces influences et ces contacts ont contribué à façonner le visage des collections publiques françaises. Cependant, si la peinture a bénéficié des recherches nourries d'A. Brejon de Lavergnée, le dessin et la gravure attendent encore leur historien. L'étude des filigranes et les documents d'archives des villes de province françaises, le dépouillement systématique des catalogues de ventes de collections du XVII ${ }^{e}$ et du XVIII ${ }^{e}$ siècle, guides de voyageurs étrangers, archives et minutes notariales d'inventaires de collections, pourraient livrer dans l'avenir quelques nouveaux secrets. Sans doute y a-t-il également beaucoup à attendre d'une étude des gravures conservées dans les fonds napolitains, et d'une observation attentive des ventes publiques françaises et étrangères.

Il fut longtemps admis en effet que l'art napolitain avait été peu goûté par les amateurs français des siècles passés, ce qui suffisait à justifier l'indigence des études consacrées, dans notre pays, à la capitale méridionale et à ses artistes. Aujourd'hui encore, les noms de de Matteis, Conca ou même Giaquinto (que B. De Dominici considérait comme le second plus grand fresquiste $\mathrm{du} \mathrm{xVIII}^{\mathrm{e}}$ siècle après G. Tiepolo), restent globalement négligés. Peu d'ouvrages et encore moins de trace dans les manuels d'histoire de l'art français suffisent à s'en persuader. Pourtant, si Naples est loin d'occuper, dans nos musées, la même place que les autres écoles italiennes, romaine, florentine ou vénitienne, quelques investigations au cœur des cabinets de dessins français révèlent les noms d'éminents "curieux", collectionneurs de dessins et tableaux napolitains, comme A. Crozat, P.-J. Mariette, C.-P. de Saint-Morys ou A.-J. Dezallier d'Argenville.

Après une relative pauvreté au XVII ${ }^{e}$ siècle, les catalogues de vente révèlent brusquement, vers 1700-1750, la présence de feuilles napolitaines en grand nombre, au moment même où les sollicitations françaises restent lettre morte (Giordano puis Solimena déclineront tour à tour l'invitation du roi, et seul de Matteis fera le déplacement jusqu'à Paris à la requête du comte d'Estrées en 1702). Si Mariette par exemple recueille plus volontiers les œuvres de L. Giordano ou F. Solimena, il ne dédaigne pas pour autant les réalisations de leurs élèves, même si l'identification des artistes est souvent incertaine et se perd fréquemment dans une vague "École de " (d'autant plus surprenante qu'artistes et collectionneurs sont alors d'exacts contemporains). Si l'aspect fantaisiste de certaines attributions, plus marqué encore au xIX siècle, trahit une connaissance assez superficielle de l'art napolitain, une étude attentive montre que ces feuilles ne sont pas 
sélectionnées au hasard mais témoignent d'un souci plus documentaire que véritablement artistique, faisant du travail de l'historien une véritable enquête à la recherche de compositions perdues, détruites ou oubliées.

Des découvertes en perspective?

19 Alors que les collections graphiques des musées publics français commencent à susciter l'intérêt des scientifiques, il y a fort à parier cependant que Naples, une fois encore, ne constituera pas la préoccupation première des études espérées. Le silence des historiens à l'égard des productions graphiques parthénopéennes traduit en effet à la fois une méconnaissance et une incertitude.

Bien des personnalités n'ont pas encore retrouvé d'identité ou de corpus véritable, et il reste difficile d'appréhender les représentants de cette ville aux mille facettes. Les recherches actuelles témoignent des confusions fort nombreuses entre les artistes et les foyers de création, mêlant sous une même attribution dessins napolitains de belle qualité, pâles copies, mains françaises et représentants d'autres foyers italiens.

21 Manquant de points de repère (absence de synthèse et profusion d'articles ponctuels), de publications récentes parfois (Solimena et Giaquinto, ou bien encore Conca, ont respectivement fait l'objet de monographies à la fin des années 1950 et au début des années 1980), l'historien français de l'art graphique napolitain se heurte souvent aux incertitudes et aux approximations. Les cabinets de dessins, encore timidement explorés, recèlent sans doute encore bien des surprises. Tardivement redécouvertes, encore peu étudiées et rarement publiées, souvent négligées, ces feuilles attendent encore leur réhabilitation.

\section{NOTES}

1. Le travail des conservateurs et des scientifiques italiens et la vitalité de Naples ont permis de réaliser des progrès considérables dans la connaissance du patrimoine graphique napolitain (début d'expositions mensuelles consacrées aux collections du musée de San Martino à partir de 1953, catalogue critique des œuvres graphiques de la Società napoletana di storia patria, par M. Causa Picone...).

2. Charles de Brosses, lettre à M. de Neuilly (24 novembre 1739).

3. L'historien commence à publier plusieurs articles consacrés à Preti, Battistello et Traversi à partir de 1913.

4. Cette rétrospective présentait, pour les Napolitains, des œuvres d'A. Belvedere, G. Bonito, C. Giaquinto, L. Giordano, F. de Mura, F. Solimena et G. Traversi.

5. Sous la direction conjointe de C. Goguel et W. Vitzthum.

6. Dessins napolitains du XVII ${ }^{e}$ siècle, Paris, palais de Tokyo, 1982-1983 et Dessins napolitains XVII ${ }^{e}$-XVIII ${ }^{e}$ siècles. Collections des musées de Naples, Paris, École nationale supérieure des beaux-arts, 1983. 


\section{AUTEUR}

\section{SOPHIE HARENT}

Sophie Harent est âgée de vingt-six ans. Conservateur au musée des beaux-arts de Quimper puis de Nancy, elle consacre ses travaux à l'histoire du dessin. Elle enseigne également à l'école du Louvre depuis octobre 2001. Cet article s'appuie sur des recherches entreprises dans le cadre d'un DEA mené sous la direction du professeur Alain Mérot, et soutenu en novembre 2000 à l'université de Paris IV-Sorbonne, consacré à Francesco Solimena et son cercle. Dessins de trois collections publiques françaises (cabinet des dessins du musée du Louvre, et des musées des beaux-arts de Dijon et d'Orléans). 